\title{
The Silent Revolution
}

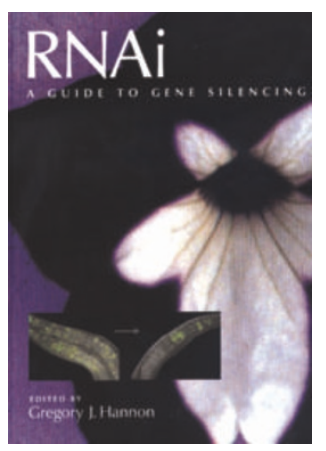

\section{RNAi: A Guide to Gene Silencing}

edited by Gregory Hannon

Cold Spring Harbor Laboratory

Press • 2003, £95/\$130

\section{Gopalakrishna Ramaswamy and Frank J. Slack}

Genome sequencing has identified thousands of novel genes with unknown function. Therefore, tools for quick and convenient gene silencing are needed - now more than ever - to determine gene function. The recently discovered phenomenon of RNA interference (RNAi) is now being used as one such tool. The term RNAi is used to describe the specific silencing of a gene with complementary doublestranded RNA (dsRNA). Silencing requires digestion of the long dsRNA into short, dsRNAs, known as small-interfering RNAs (siRNAs). These siRNAs enter a nuclease called the RNA induced silencing complex (RISC) and act to guide RISC to the corresponding native mRNA for degradation. In many species, RNAi is a naturally occurring process that seems to regulate gene expression and defend against invasion by viruses and transposons.

Now, RNAi is being used in the laboratory as a method to specifically reduce product levels of almost any gene of interest. The convenience and low cost of RNAi has opened up the door to large-scale, high-throughput screens on a scale only dreamed of a few years ago. In addition, this technology is also being tested for therapeutic purposes against cancers and viral infections. Given recent advances both in applying RNAi-based technology and in understanding how small RNAs regulate cellular pathways, a book such as RNAi: A Guide to Gene Silencing, edited by Gregory Hannon - that covers the history and current state of understanding of small RNA-based processes should prove to be interesting to many.

RNAi: A Guide to Gene Silencing consists of eighteen chapters, written by a selection of people with diverse expertise in the field of RNAmediated silencing. The initial chapters are dedicated to an overview of the discovery and mechanistic basis of RNAi, and related silencing phenomena, including post-transcriptional gene silencing, transcriptional gene silencing and co-suppression. Gregory Hannon and Phillip

Gopalakrishna Ramaswamy and Frank J. Slack are in the Department of Molecular, Cellular and Developmental Biology, Yale University, P.O. Box 208103, New Haven, CT 06520, USA.

e-mail:frank.slack@yale.edu orgopalakrishna.ramaswamy@yale.edu
Zamore then discuss the biogenesis and mechanistic details of RNAi. This chapter not only explains how RNAi functions in different organisms, but also how it is mechanistically related to miRNAs and other small-RNA-mediated pathways. This is followed by detailed chapters explaining silencing mechanisms that depend on the RNAi machinery, such as co-suppression in plants and animals and the regulation of transposable elements and heterochromatin in plants, animals and fungi. These chapters highlight well the differences between transcriptional and post-transcriptional silencing mediated by small RNAs. RNAi has been demonstrated in many organisms, and individual chapters dedicated to those in which RNAi is extensively studied are particularly useful. These chapters provide specialized protocols that are useful to those interested in adapting this technology to their organism of interest. In addition, the development of RNAi libraries has been important for genome-wide screening and functional genomic studies. Accordingly, several chapters explain how RNAi - and the related technique of virus-induced gene silencing (VIGS) — could be used for large-scale screens in both model organisms and mammalian cells alike. Although long dsRNA can initiate RNAi in plants, Drosophila and C. elegans, the use of dsRNAs of more than 30 base pairs in length leads to non-specific translational arrest and apoptosis in somatic mammalian cells. An important advance in our understanding of RNAi in somatic mammalian cells stemmed from the discovery by Thomas Tuschl and others that shorter siRNAs (21-nucleotide duplexes) can lead to the specific degradation of cognate mRNAs. Multiple chapters are dedicated to describing these findings and the more recent creation of DNA vectors for transient or stable expression of siRNAs or smallhairpin RNAs (shRNAs) in a range of organisms and cell lines. Although the book makes a strong effort to cover multiple facets of RNAi and the diversity of organisms utilizing RNA-silencing, omissions in a few areas are noticeable. There is an excellent chapter on RNAi in C. elegans by Ronald Plasterk and colleagues, but the genetics of RNAi in general are not as thoroughly addressed. Given their potential significance as chromosomally encoded regulatory molecules, it would also have been good to see a specific chapter on microRNAs, perhaps highlighting similarities and differences with siRNAs. Additional details on specific rules for designing effective siRNAs, and perhaps chapters on the use of RNAi in pharmaceutical and clinical settings, might also have been useful. Given that there are multiple authors, the book does suffer slightly from redundant information, but overall this is a small price to pay for having all the information in one book.

This book provides a useful and relatively comprehensive summary of progress in the field, and it is likely that every biologist will find something of interest. While experts in this field will be able to update their knowledge on RNAi and its application (for example, its use in high-throughput screening), the book should also serve as a useful introduction to the world of small RNAs for the non-expert. An added bonus is the inclusion of many protocols that explain the details of key RNAi experiments that once again should prove useful to the expert and non-expert alike. Finally, given the rapid advance in this field, it is likely that another such book will be needed in the near future. 